\title{
Chapter 2 \\ Supramolecular Congo Red as Specific Ligand of Antibodies Engaged in Immune Complex
}

\author{
Anna Jagusiak, Joanna Rybarska, Barbara Piekarska, Barbara Stopa, \\ and Leszek Konieczny
}

\begin{abstract}
Supramolecular Congo red has been used to validate long-lasting theories regarding intramolecular signaling in antibodies and its relation to activation of the complement system. Strong enhancement of antigen-antibody complexation resulting from the binding of supramolecular ligands enables also polyclonal antibodies having intermediate affinity to trigger complement cascade apart of high affinity antibody fraction. This would not have been possible in the absence of Congo red. The property of antibodies provides specifically their ability to trigger the complement system allowed when sufficient structural strain is produced by antigen complexation provides an evidence of intramolecular signaling.

The selective complexation of supramolecular ligands with antibodies engaged in immune complexes enables their using as carriers of drugs in immunotargeting system.
\end{abstract}

Keywords Intramolecular immunological signal $\bullet$ Complement activation $\bullet$ IgG V domain stability $\bullet \mathrm{N}$-terminal fragment $\bullet$ Enhancement of antigen binding $\bullet$ Congo red as carrier of drugs $\bullet$ Immunotargeting system • Congo red selective complexation of antibodies in immune complexes

Self-associating organic molecules which form ribbonlike micellar structures may, owing to their structural characteristics, penetrate inside proteins and form stable complexes. Such penetration is possible in areas of the protein which have been destabilized, either temporarily or permanently - such as antibody/antigen complexes. Since Congo red (CR) has been used in research as the most typical

\footnotetext{
A. Jagusiak $(凹) \bullet$ J. Rybarska $\bullet$ B. Piekarska $\bullet$ B. Stopa $\bullet$ L. Konieczny

Chair of Medical Biochemistry, Jagiellonian University - Medical College,

Kopernika 7, 31-034 Krakow, Poland

e-mail: anna.jagusiak@uj.edu.pl; mbstylin@cyf-kr.edu.pl; mbpiekar@cyf-kr.edu.pl;

barbara.stopa@uj.edu.pl; mbkoniec@cyf-kr.edu.pl
} 
supramolecular protein ligand, the presented experiments and analysis also focus on this particular dye. CR binds strongly to antibodies, enabling us to study (among others) intramolecular signaling related to complement system activation. What is more, the mutual affinity of CR and immune complexes paves the way towards immunotargeting, i.e. targeted delivery of drugs. This is due to the fact that supramolecular CR - a micelle-like structure - may intercalate foreign bodies, including drug molecules. Congo red does not react with free antibodies - it is only capable of binding to antibody/antigen complexes where structure of antibody undergoes some alteration due to interaction with the antigen. Any potential drugintercalated into the CR micelle can thus be delivered to an area where the antigen is plentiful, ensuring targeted action. This chapter discusses the presented topics in detail.

\subsection{Looking for Evidence of Postulated Intra-molecular Immunological Signaling}

Once the structure of immunoglobulins has been divined, it soon became clear that their Fab and Fc fragments play differing roles in the process of triggering immunological response. While the Fab fragment selectively binds to the antigen, the Fc fragment - separated by a hinge - appears to be involved in triggering complement system activation through complexation of the Clq subcomponent. Notably, the Fab-antigen interaction is independent of $\mathrm{Fc}$ and proceeds even when the $\mathrm{Fc}$ fragment has been removed by digestion $[1,2]$.

The complement system is a collection of proteins which attack and destroy cells recognized as alien by the immune complex. Strict control over this mechanism is critical for homeostasis and therefore represents an important study subject in medical research. In accordance with prevalent views, such control is maintained by intramolecular rearrangements which carry information from $\mathrm{Fab}$ to $\mathrm{Fc}$, and then onwards to C1q (Fig. 2.1).

Fig. 2.1 Schematic depiction of the intramolecular signaling pathway inside the antibody (dashed line)

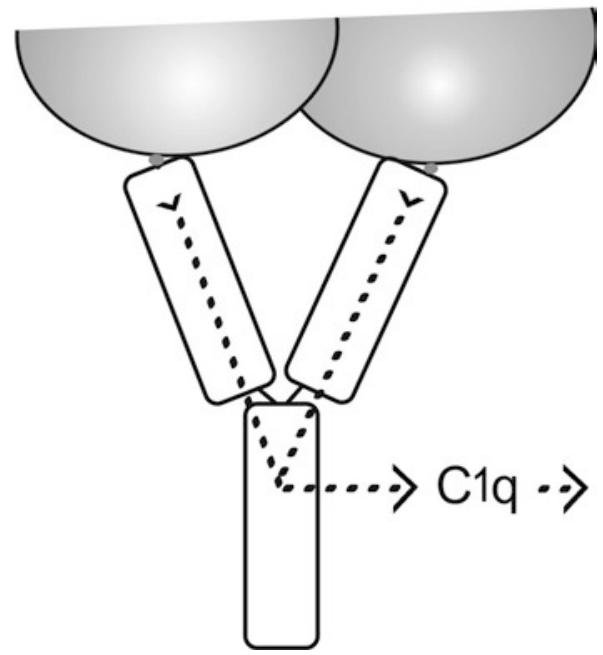


Nevertheless, despite significant effort by many leading researchers, the specifics of this mechanism have proven exceedingly difficult to elucidate and some uncertainties persist. In attempting to explain intramolecular signaling, analysts initially focused on the hinge region which links Fab and Fc. Experimental data indicates that subclasses of immunoglobulins which differ with respect to the composition of this hinge region also exhibit variable efficiency of Fab-to-Fc signal transmission, and moreover that reduction of the disulfide bond in the hinge region prevents successful activation of the complement system [3-5]. In turn, some attention was directed towards structural strain in the antibody molecule, produced by antigen binding and regarded as a possible signal carrier. This view is embodied in the socalled distortive mechanism theory. Another competing theory proposed an "all or none" switching mechanism, i.e. an allosteric model based on the assumption that immunoglobulins are, in fact, allosteric [6].

Since none of the presented models succeeded in providing a satisfactory explanation, further analysis was needed. Some researchers noted the fact that, under ordinary circumstances, the formation of an active immune complex involves many different antibodies, and that complement activation requires local concentration of Fc fragments. This so-called associative model appeared to explain the signaling puzzle to a sufficient degree, particularly given the lack of evidence favoring intramolecular signaling [7, 8]. Earlier theories were swept aside and the issue appeared solved. This situation persisted for many years, until scientists learned how to produce monoclonal antibodies via crystallization of Fab fragments cleaved from the IgG molecule, and formulated new analysis protocols based on the use of small antigens (haptens) [9-11]. Surprisingly, these studies produced little in the way of useful results. Structural changes appeared small, even negligible - again suggesting that intramolecular signaling must somehow involve torsional effects, which emerge only when the antigen is bound to a complete, two-arm antibody.

Spectacular progress in genetics achieved in the 1980s, particularly the ability to synthesize arbitrarily modified antibodies, brought new hope of understanding the purported intramolecular signaling mechanism. Still however, despite some focus on the interaction of $\mathrm{CH}_{1}$ and $\mathrm{CH}_{2}$ domains, the problem of signal remained practically unsolved [12-14].

Our research group decided to attack the problem through chemical recombination of antibodies by digestion, reduction and re-joining of immunoglobulin fragments solely by disulfide bonds. The goal was to determine whether this kind of modified structure would retain the ability to carry the signal to Fc, despite major alterations in the hinge region. This process is illustrated in Fig. 2.2.

While the "full" two-arm molecule constructed from free Fab and Fc fragments exhibited complement activation potential (to a limited degree), its one-arm equivalent proved entirely inert. This suggested that even a deficient antibody may transmit the signal, if only suitable conditions exist for structural strain to emerge. Nevertheless, the participation of the hinge region in signal transmission remained a mystery $[3,15,16]$. 


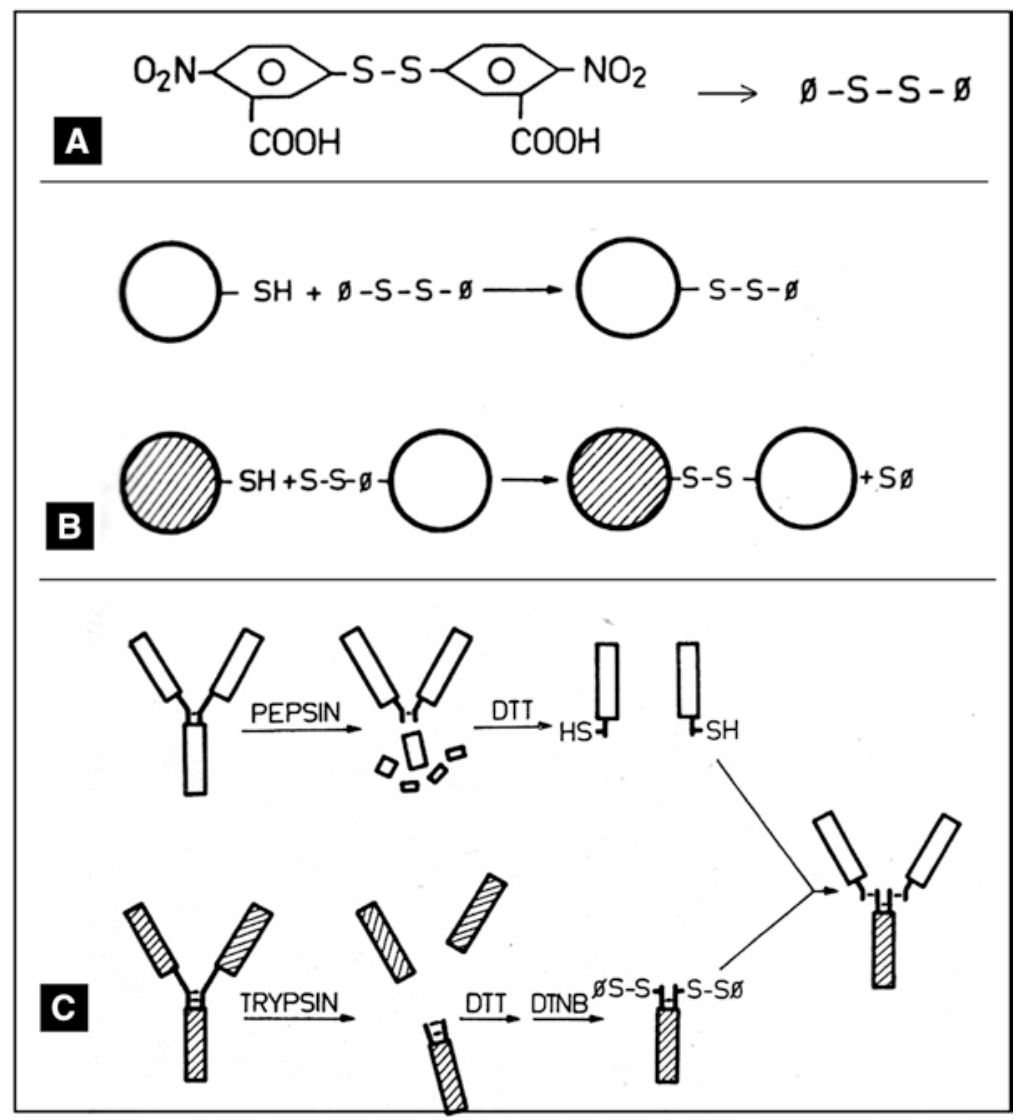

Fig. 2.2 Schematic view: (A and B) Controlled formation of linking disulfide bonds. (C) Production of recombinant IgG

\subsection{Evidences of Intramolecular Signaling Supplied by Using Congo Red}

A whole new approach to the problem was enabled by the use of CR, based on our team's original concept. While CR had long been known as a useful amyloid stain, its interaction with amyloids was explained as individual molecules attaching themselves to specific binding sites which recognize the dye. In contrast, our study revealed that $\mathrm{CR}$ may form complexes with a wide variety of proteins and that it does so as a supramolecular ligand - i.e. a distinct structure consisting of many associated dye molecules acting as a single unit [17-25]. 
Fig. 2.3 V domain of the L chain lambda, with its hightlighted N-terminal fragment covering gap created by its removal. Space filling model

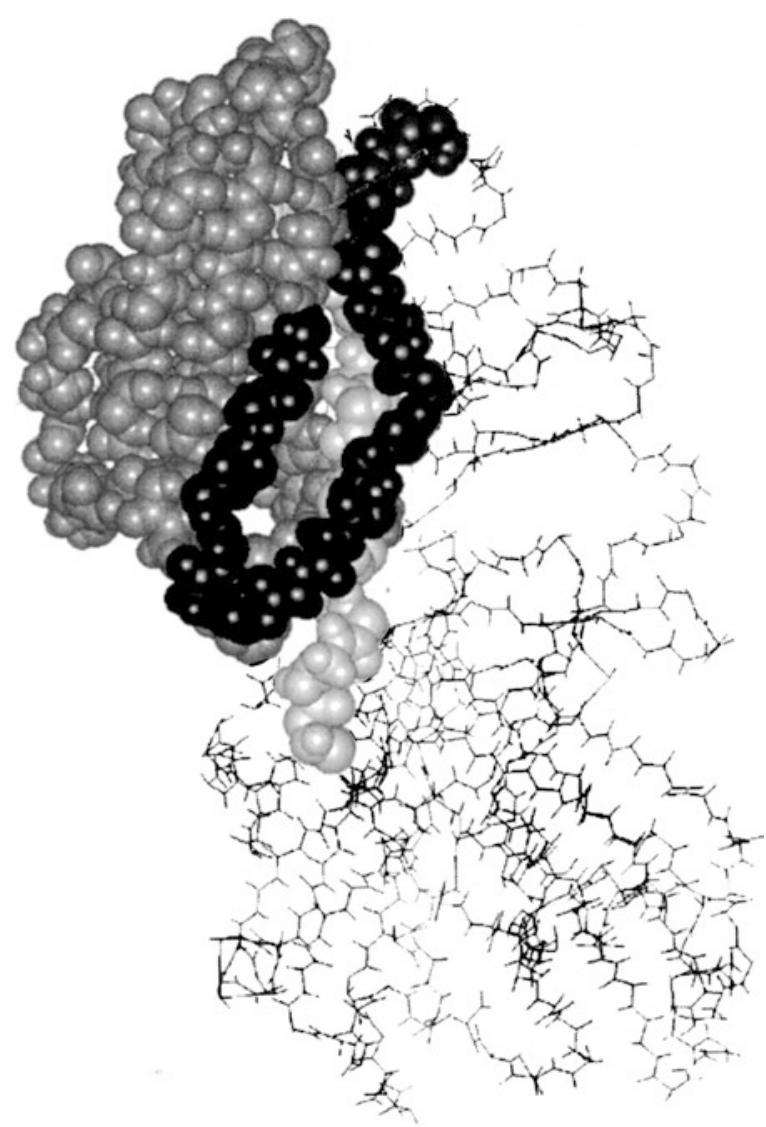

An important breakthrough occurred when CR was found to interact with immune complexes, but not with free antibodies. This phenomenon shed new light on the intramolecular signaling pathways leading to complement system activation. Such selective binding suggested that CR is capable of anchoring itself in the V domain of the antibody, which also happens to be the site of the greatest structural strain resulting from antigen complexation. Furthermore, the $\mathrm{V}$ domain also houses the N-terminal fragment, which, by default, is relatively unstable (Fig. 2.3) [26-28].

Confirmation of this theory was provided by analyzing the complexation potential of CR vs. light chain dimers progressively destabilized through heating or increased concentrations of the dye. The displacement of the N-terminal fragment from its packing locus "opens up" the V domain, enabling the supramolecular dye to penetrate and anchor itself in its interior. Under experimental conditions, the first complexes to emerge involve ligands composed of four molecules. As the dye concentration increases, the ligand may grow to include up to eight dye molecules (Fig. 2.4A, B respectively). This is evidenced by electrophoresis, where larger com- 

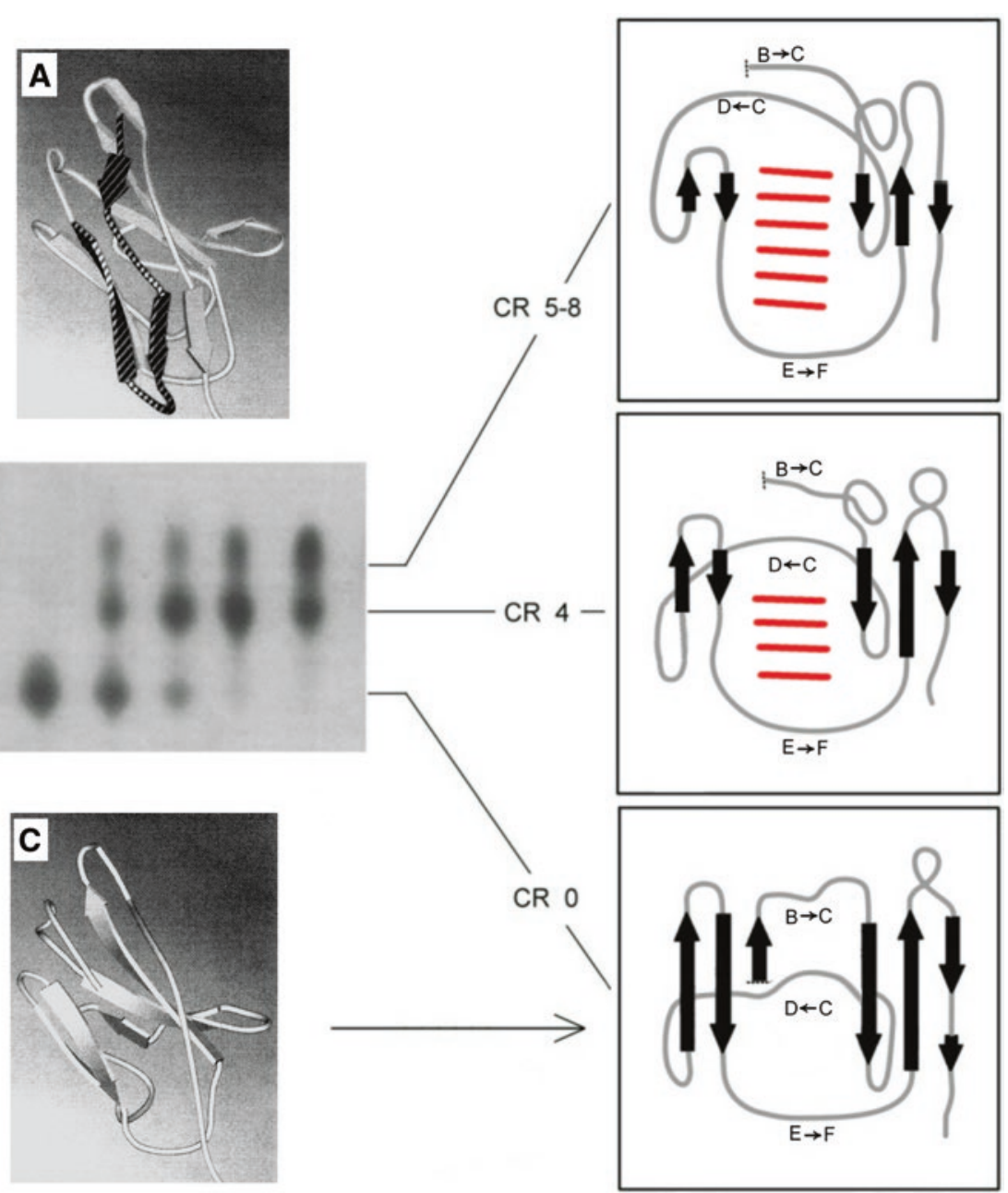

Fig. 2.4 Complexation of $L$ chain dimer with CR (A) Light chain V domain. (B) Thermally generated complexes of $\mathrm{CR}$ with the $\mathrm{L}$ chain $\mathrm{V}$ domain presented by corresponding models. (C) V domain deprived of its $\mathrm{N}$-terminal fragment through digestion. Agarose electrophoresis of $\mathrm{L}$ chains induced to form complexes with CR upon the stepwise increasing temperature

plexes migrate faster due to their greater charge (contributed by CR). In each case, the trigger for complexation appears to be the N-terminal fragment, which is displaced from its packing locus and replaced by the supramolecular ligand. The displaced N-terminal fragment subsequently becomes susceptible to digestion [26, 29-31].

$\mathrm{CR} /$ light chain complexation may be accelerated by heating, which further perturbs the N-terminal fragment. In contrast, when dealing with immune complexes, complexation appears to be induced by structural strain resulting from antigen bind- 


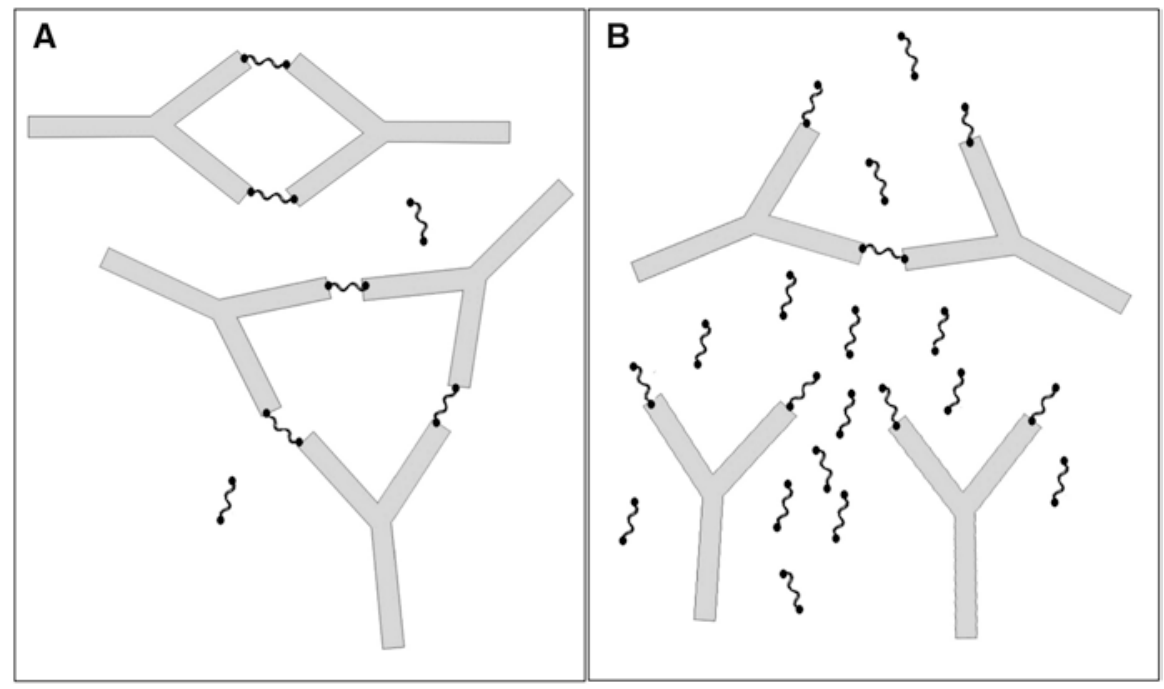

Fig. 2.5 Immune complexation of antibodies linked by binary haptens (A) and breakage of immune complexes caused by overabundance of hapten particles $(\mathbf{B})$

ing. This phenomenon, however, only emerges in complete two-arm antibodies, attaching themselves to antigen determinants located randomly on the cell surface. Neither isolated Fab fragments nor their dimers are capable of binding CR, even in their complexed state. This proves that the antibody-antigen reaction is not directly responsible for the affinity to $\mathrm{CR}$, and that dye complexation requires structural strain in the antibody molecule [26, 29-31].

CR is complexed by whole antibodies in complex with haptens, but only when they are fixed on a solid surface, i.e. under conditions which lead to structural strain in bivalent antibodies.

CR also binds to anti-TNP antibodies linked by a hapten if the hapten itself is bivalent and creates strain in the antibodies it links (e.g. oxidized glutathione with amino groups substituted with TNP - Fig. 2.5).

This can be confirmed under electrophoresis, since the antibodies bound by the binary hapten form immune complexes, become soluble and migrate more rapidly when treated with CR. Their number grows as the concentration of the hapten increases, eventually reaching a maximum beyond which a falloff is expected (Fig. 2.6) - due to the fact that when the hapten is overly abundant, it becomes monovalent and therefore produces no strain in the attached antibodies (Fig. 2.5) [32].

The link between CR and immune complexes exhibits one more remarkable property: it turns out that complexation of CRgreatly enhances theantigen/ antibodycomplexation capabilities. This is evidenced by a significant increase in the fraction of antibodies involved in immune complexes, especially in relation to low-affinity antibodies which are naturally present in the polyclonal serum. It should be noted that the serum contains many different types of antibodies with 


\section{A}

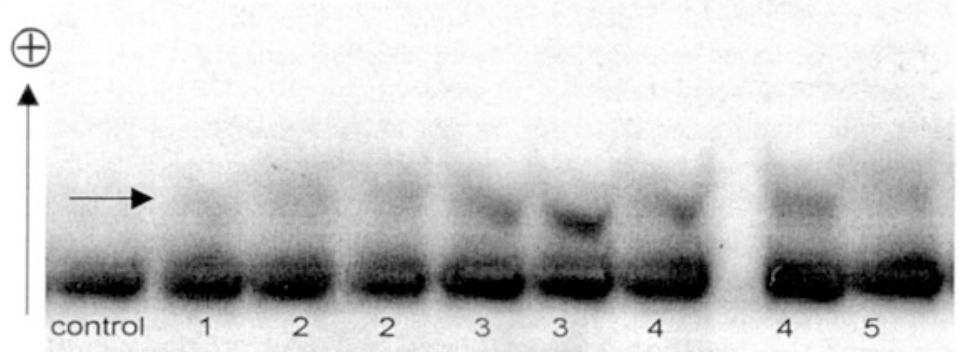

B

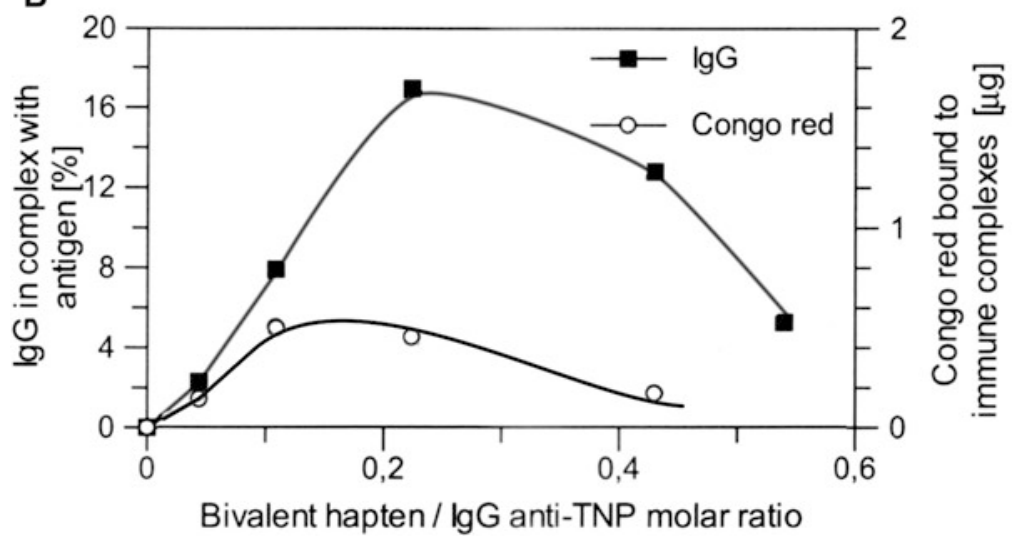

Fig. 2.6 Experimental evidence of formation soluble complexes with CR by binary hapten-linked antibodies (as depicted in Fig. 2.5). Left to right: increasing hapten concentrations. The mobile fraction (indicated by the arrow) comprises soluble immune complexes which have gained the ability to bind CR (Reproduction by permission - J Physiology and Pharmacology)

varying degrees of affinity - due to the inherent randomness in the antibody synthesis process. Low-affinity antibodies cannot form stable immune complexes and are washed out in the absence of CR. When the dye is present, their ability to bind antigens increases and the number of immune complexes per unit of volume grows (Fig. 2.7 A, B).

At this point it would be useful to determine the mechanism which drives increased complexation capabilities in the presence of CR, and also to find out whether such upregulation is accompanied by the corresponding increase in complement system activity (which would prove the existence of an intramolecular signal).

The assumption that, by binding its natural biological ligand, the protein undergoes structural rearrangement which favors penetration and complexation of supramolecular dye would explain why the ligand cannot be easily released once the protein-dye complex has formed. This phenomenon appears actual in situations 

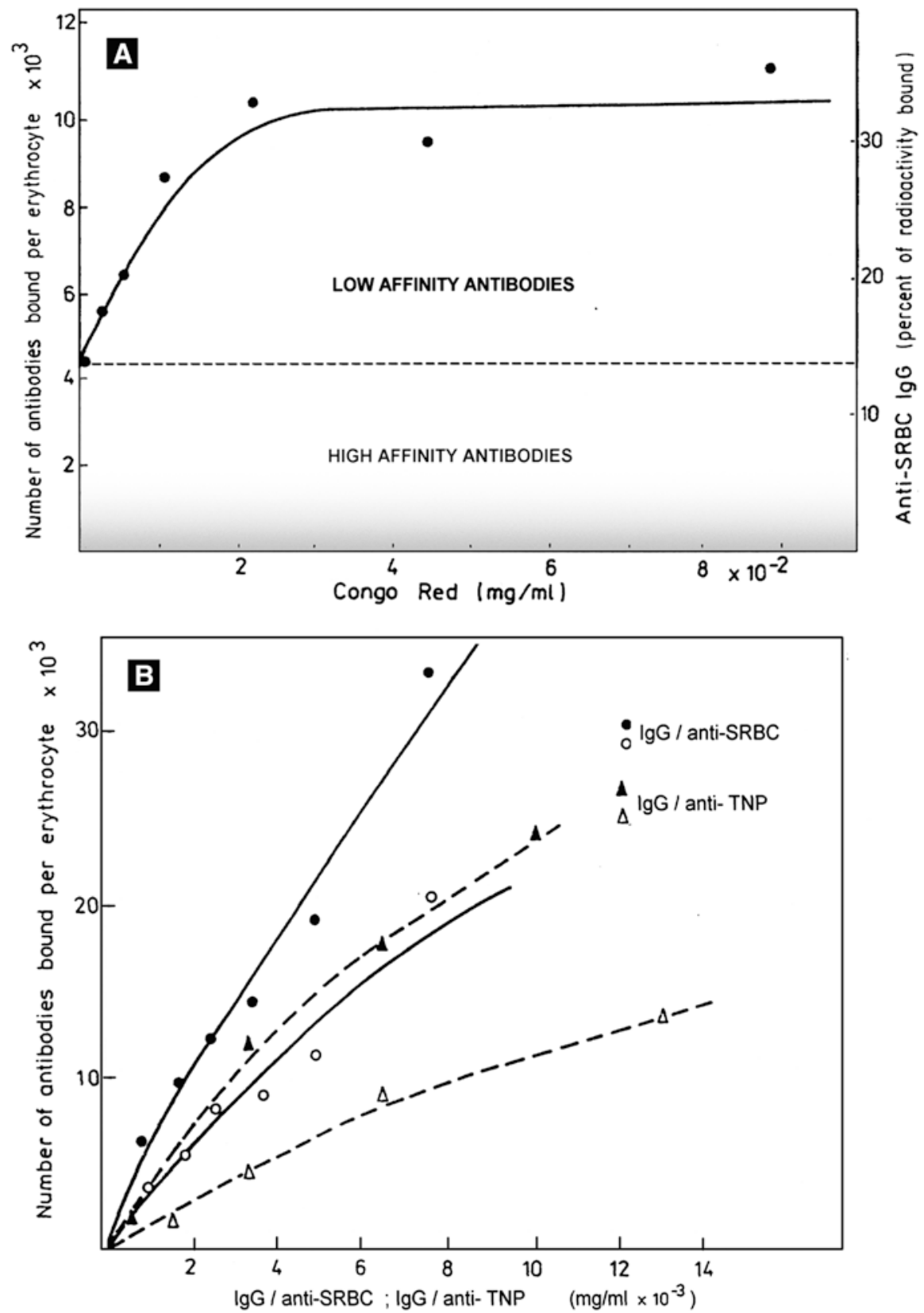

Fig. 2.7 Profiles presenting: (A) Increase in the quantity of erythrocyte-agglutinating antibodies under increasing concentrations of CR. High-affinity antibodies are capable of agglutinating cells in the absence of the dye. Anti-SRBC antibodies complex tagged by ${ }^{125}$ I. High affinity antibodies fraction non removable by washing. Shadowed area - fraction of highest affinity. (B) Enhancement of antibody/antigen interaction by CR, proving that the effect is independent of the type of antigen and specificity of the antibody (Reproduction by permission - Archivum Immunologiae and Therapie Experimentalis) 
where irreversibility is finally expected - immune complexation, C1q binding etc. On the other hand, the same phenomenon would tend to inhibit the action of enzymes where the ligand must be released following catalysis. Indeed, such inhibition has been confirmed in the scope of complement activation which depends on the action of convertases [33].

The observed enhancement of antibody/antigen complexation capabilities may also be due to another factor: increased flexibility of the $\mathrm{V}$ domain, caused by penetration of a large noncovalently stabilized ligand to packing cavity of the replaced $\mathrm{N}$-terminal fragment, bestowing greater internal mobility upon the domain (particularly its CDR loops) and therefore enabling them to align themselves to the antigen with greater accuracy [34].

The discovery and subsequent theoretical study of antigen complexation enhancement triggered by CR creates new possibilities with regard to analysis of intramolecular signal leading to complement system activation - assuming that such signal exists. Due to inhibition of convertase (and therefore of the complement system) by CR, measured as the efficiency of hemolysis, the signal transfer stage (immune complex/C1q) has been separated from the remainder of the activation cascade, including convertase. This reveals activation potential, since both the immune complex and the subsequent complex with $\mathrm{C} 1 \mathrm{q}$ are insoluble and may therefore be separated from excess CR by washing, then combined with the remaining components of the complement system, thus preventing undesirable inhibition. To this end we have employed a commercial-grade C1q reagent (QUIDEL USA) and, separately, a serum containing complement system components but deprived of C1q (QUIDEL USA).

Once the excess dye has been washed out, the remaining insoluble complexes (immune complex and immune-C1q complex) prove capable of activating the complement system, triggering hemolysis in C1q-deprived serum. Figure 2.8 presents the results of this experiment. Confirmation of complement system activation reveals the role of $\mathrm{CR}$ in the process and confirms the presence of intramolecular signaling.

The immune complex (agglutinate) binds antibodies with varying affinity for red cells which participate in the immune response (SRBC/anti-SRBC). Weak (lowaffinity) antibodies are quickly washed out in the absence of CR. The remaining complexes contain antibodies with strong or moderate affinity. The introduction of CR stabilizes the immune complexes formed by weak antibodies, allowing them to remain in the agglutinate. Nevertheless, such antibodies remain incapable of triggering hemolysis even when CR is present. Their properties may be studied by analyzing wash-out samples. Of course, antibodies which resist washing out are also characterized by variable affinity: the group includes strong (high-affinity) antibodies which do not require CR to form stable complexes and trigger hemolysis, but also weak (low-affinity) antibodies stabilized by CR but still unable to trigger hemolysis. The specific affinity threshold established by the wash-out procedure is somewhat arbitrary and depends on a number of conditions. It is assumed that CR account for approximately $50 \%$ of the washout-resistant pool. Interestingly, this group contains also antibodies which are unable to trigger complement activation, 


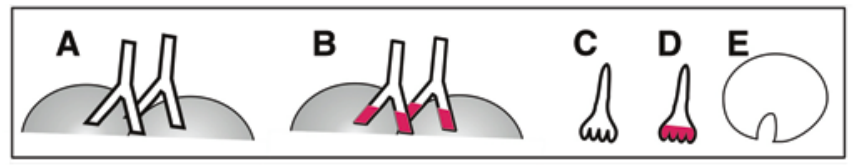

A N T I B O D I E S

HEMOLYSIS \%

ENGAGED IN

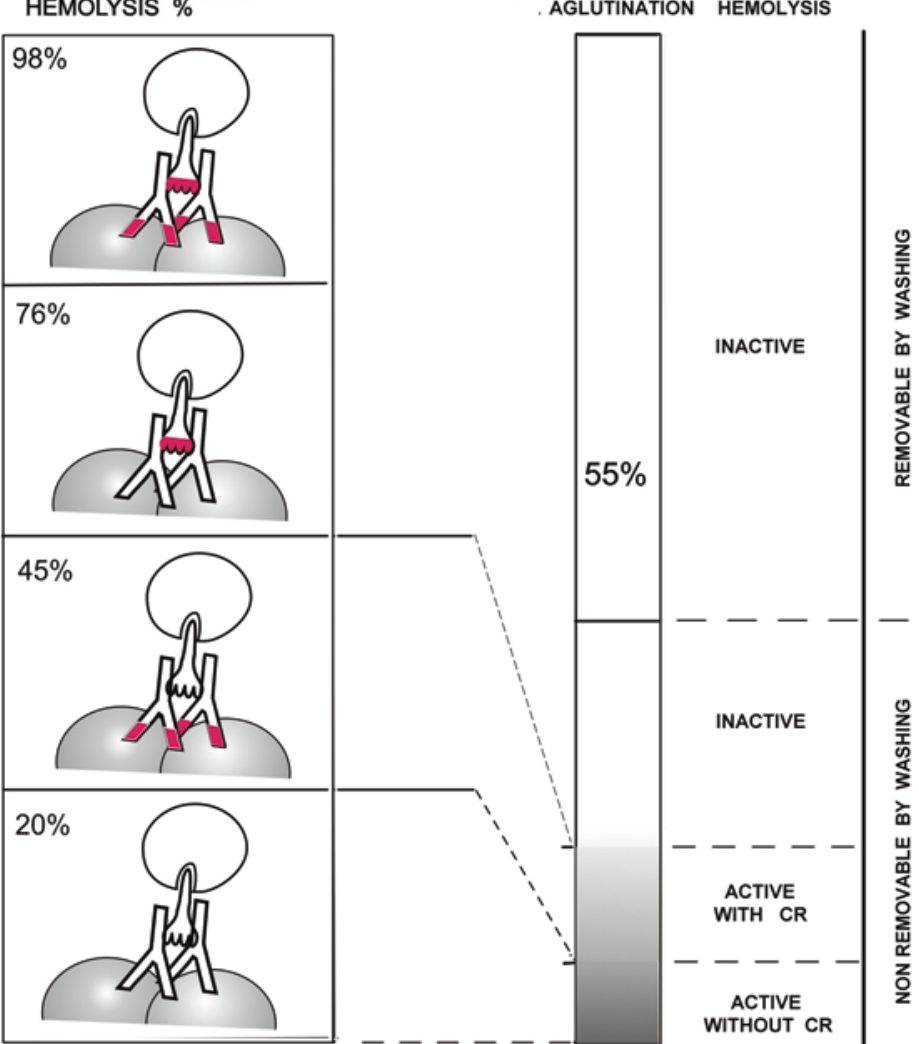

Fig. 2.8 Role of CR in amplifying the complement activation signal. Model view of the process (left-hand column). The efficiency of erythrocyte hemolysis by the complement system depends on $\mathrm{CR}$ activation of the signaling pathway. Selective action of CR upon successive components of the signal pathway has been marked in red. $A$-immune complex (anti-SRBC antibodies not treated with CR); $B$ - antibodies selectively activated by CR; $C-$ C1q; $D-$ C1q selectively activated by $\mathrm{CR} ; E$ - serum containing all components of the complement system except C1q. Participation of anti-SRBC polyclonal serum antibodies in agglutination and hemolysis is shown on the right

but which gain this ability by interacting with CR. This suggests that a sufficiently powerful intramolecular signal may only be generated by "strong" antibodies which incur significant structural strain when binding the antigen. This natural threshold appears evolutionarily conditioned to prevent accidental thus potentially dangerous activation of the complement system [35-38]. 


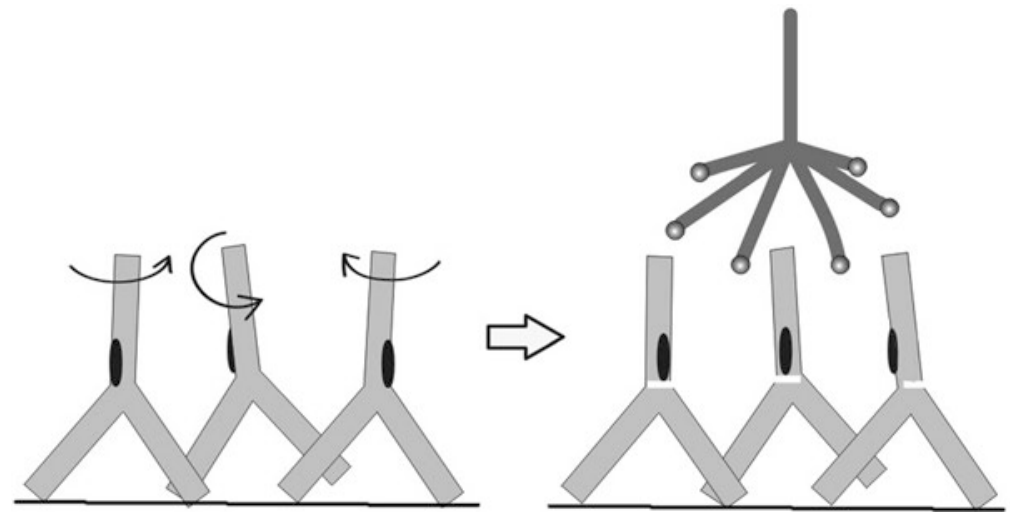

Fig. 2.9 Complexed antibodies showing random placement and orientation of $\mathrm{Fc}$ fragments. Arrows indicate the need for rotation, facilitating contact with C1q. This is enabled by intramolecular signaling which increases the mobility of $\mathrm{Fc}$ fragments

While CR helps explains the signaling mechanism, the origin of the signal itself remains an open question. Since it seems improbable for both $\mathrm{V}$ domains ( $\mathrm{VH}$ and $\mathrm{VL})$ to react to structural strain in the same way, antigen complexation induces torsional stress in the Fab fragment [39]. The resulting rotation uncouples Fc from Fab and allows the former to bind to C1q. This is important, since in the immune complex both the antibodies and their Fc fragments are oriented randomly, which would otherwise hamper complexation of $\mathrm{C} 1 \mathrm{q}$. The uncoupling provides $\mathrm{Fc}$ with rotational freedom and enables the link to be established easier (Fig. 2.9).

In this way, the use of a supramolecular dye approaches understanding both the function and the purpose of intramolecular signaling.

\subsection{Application of Congo Red for Immunotargeting}

The fact that CR selectively binds to antibody/antigen complexes creates an interesting opportunity with regard to targeted drug delivery. CR is not only capable of recognizing the immune complex, but - owing to its supramolecular nature - intercalate various drug particles, acting then as a carrier. Such intercalation should not be regarded as simple mixing of dye and drug molecules - it is more akin to solvation, which involves close interaction between the solvent and the solute. If the solute is structurally flat and presents a planar arrangement of aromatic rings, it can be easily intercalated into the CR micelle. This effect is further enhanced if the solute is positively charged (Fig. 2.10) [34, 40, 41].

The model presented in this chapter comprises CR and rhodamin B (Fig. 2.10). Interaction between both dyes is evidenced by tracking the release of rhodamine $\mathrm{B}$ from a dialysis bag in the absence of CR and in a system where both dyes are combined via intercalation. It is readily evident that rhodamine B - itself a supramolecu- 


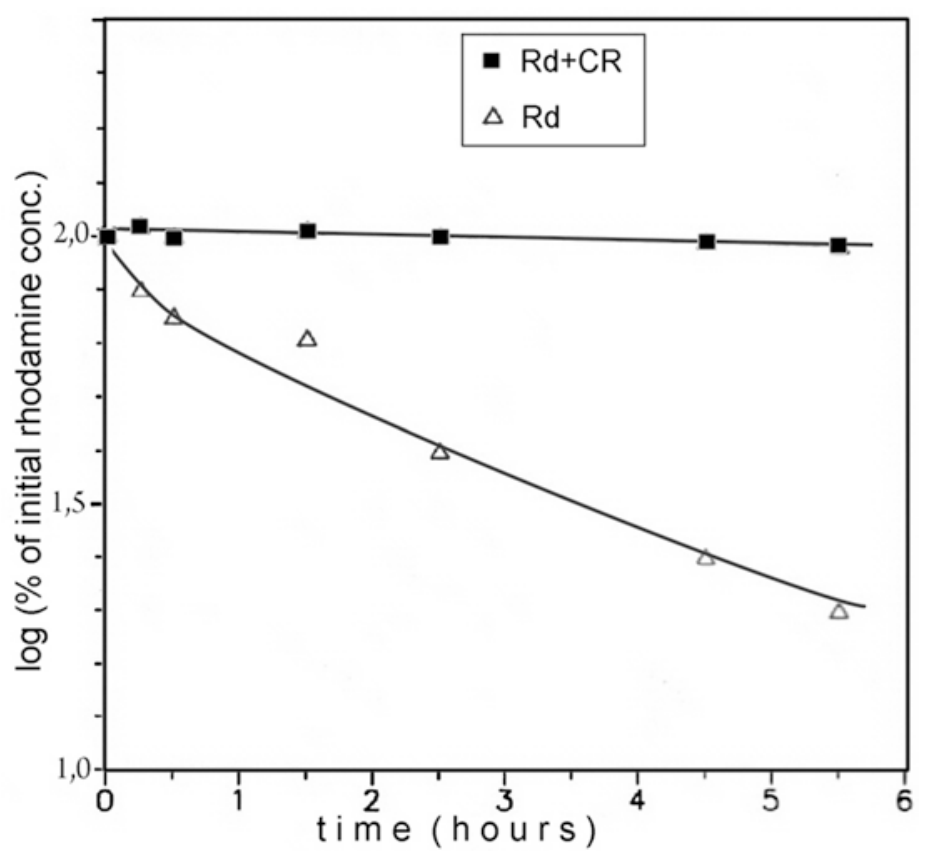

Fig. 2.10 Evidenced by dialysis arresting of rhodamine B (Rd) by supramolecular CR (intercalation), compared to progressive reduction in the concentration of free rhodamine $\mathrm{B}$

lar system, but with weaker self-association tendencies - becomes nearly impervious to dialysis when $\mathrm{CR}$ is present. Additional complexes comprising supramolecular dyes and various foreign molecules, analyzed using the dialysis method, are listed in table shown in Fig. 2.11, and are compared to the model CR/rhodamine B complex. Where analysis suggests similar stability of both complexes, a value of 1 is listed. Figure 2.11 presents selected compounds which form co-micellar structures with CR, listing their stability [42]. It seems clear that both the spatial structure and electric charge play an important role.

To further confirm the carrier hypothesis, our analysis focused on a specific immune complex, i.e. agglutination of sheep erythrocytes capable of binding supramolecular ligands. Figure 2.12 presents a visualization of the agglutinate, following addition and subsequent washing out of CR with intercalated rhodamine B [43]. Strong fluorescence of rhodamine B overcomes CR absorption and can be clearly seen along boundaries of agglutinated erythrocytes. This proves that the antibodies involved in agglutination (immune complexes) are bound to the $\mathrm{CR} /$ rhodamine $\mathrm{B}$ aggregate. Notably, CR does not react with free antibodies - only antibodies engaged in immune complexation can bind the dye. In vitro analysis therefore provides evidence of the ability of supramolecular dye to serve as a vehicle for targeted delivery of drugs. 


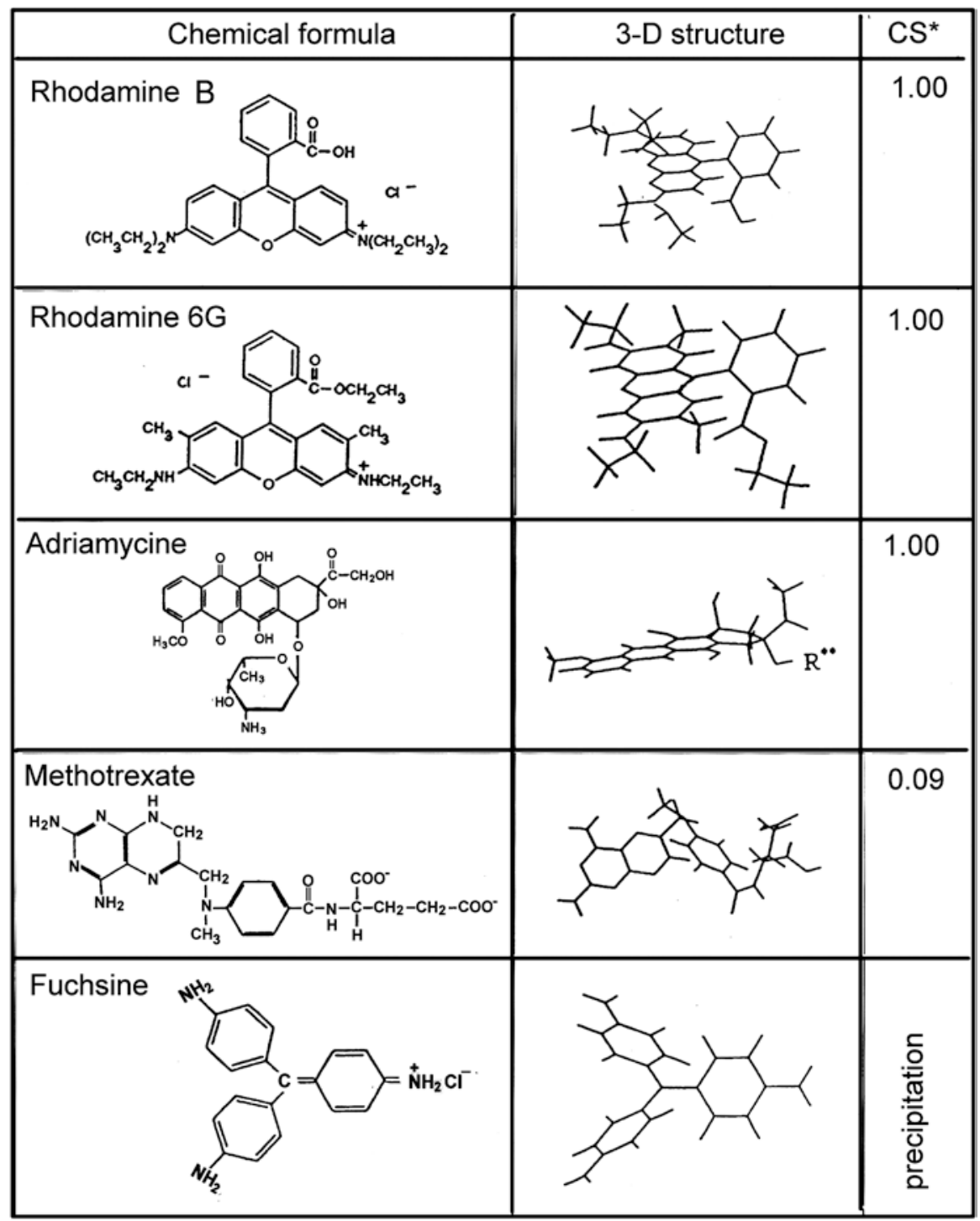

Fig. 2.11 Examples of structures readily intercalated by supramolecular CR due to their planar structure and/or positive charge. Complexation tendency ranged 0-1. (Reproduction by permission - J Physiology and Pharmacology) 

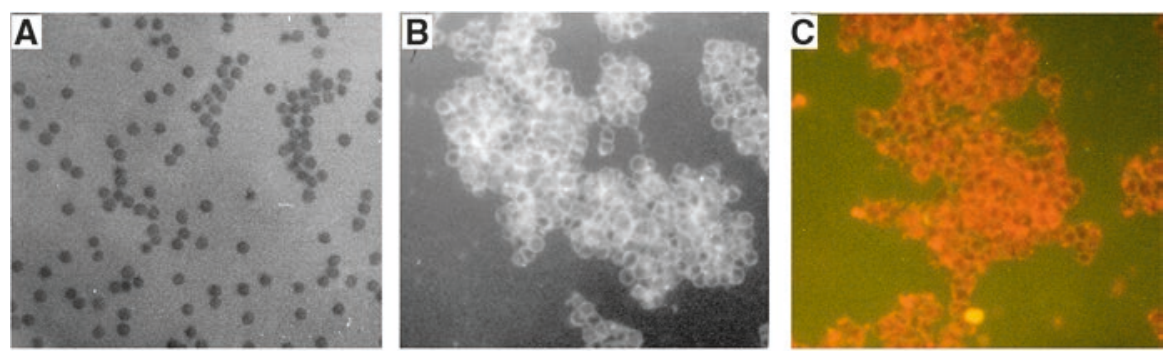

Fig. 2.12 Agglutination in the SRBC/anti-SRBC system produced by antibodies in complex with CR and rhodamine B intercalated. (A) not agglutinated red cells - control sample; (B and $\mathbf{C})$ agglutinated red cells - UV light (Reproduction by permission - Folia Histochemica et Cytobiologica)

It should be noted that in vitro test results do not always translate into similar outcomes in vivo. Successful use of supramolecular drug carriers in living organisms remains a complex problem due to undesirable reactions with serum proteins, particularly albumin. To determine whether the presented method is feasible, an Arthus reaction has been triggered in a rabbit host - i.e. the TNP antigen (ghosts of rabbit red blood cells conjugated with human $\operatorname{IgG}$ ) was injected into the earlobe of an sensitized rabbit, producing local inflammation caused by aggregation of immune complexes. The other ear was subsequently injected with $2.5 \mathrm{ml} 5 \mathrm{mg} / \mathrm{ml}$ ) isotonic steryl CR solution. The ear where the Arthus reaction had originally been triggered was then backlit for photographic documentation (the rabbit's thin earlobe is easily penetrated by visible light, simplifying the process). Unfortunately, the unaided human eye is unable to distinguish between CR and hemoglobin - the blood present in the vessels in earlobe produces an color image similar to CR. Enhanced visibility of small blood vessels suggests that an inflammatory process is ongoing, which further hampers attempts to visualize dye accumulations. Effective analysis therefore required the use of specially prepared spectroscopic filters (the spectra of CR and hemoglobin differ somewhat).

Figure 2.13 presents the backlit tissue fragment with spectroscopic filters applied. The modified color scale enables us to easily distinguish the dye and hemoglobin (filter spectra are also presented, showing which wavelengths have been blocked). As expected, the dye is attracted to the antigen injection site, where the immune complex can be found. The experiment also highlights the kinetic characteristics of CR absorption and subsequent removal, showing how the proposed transport system may function in practice (Fig. 2.14). 

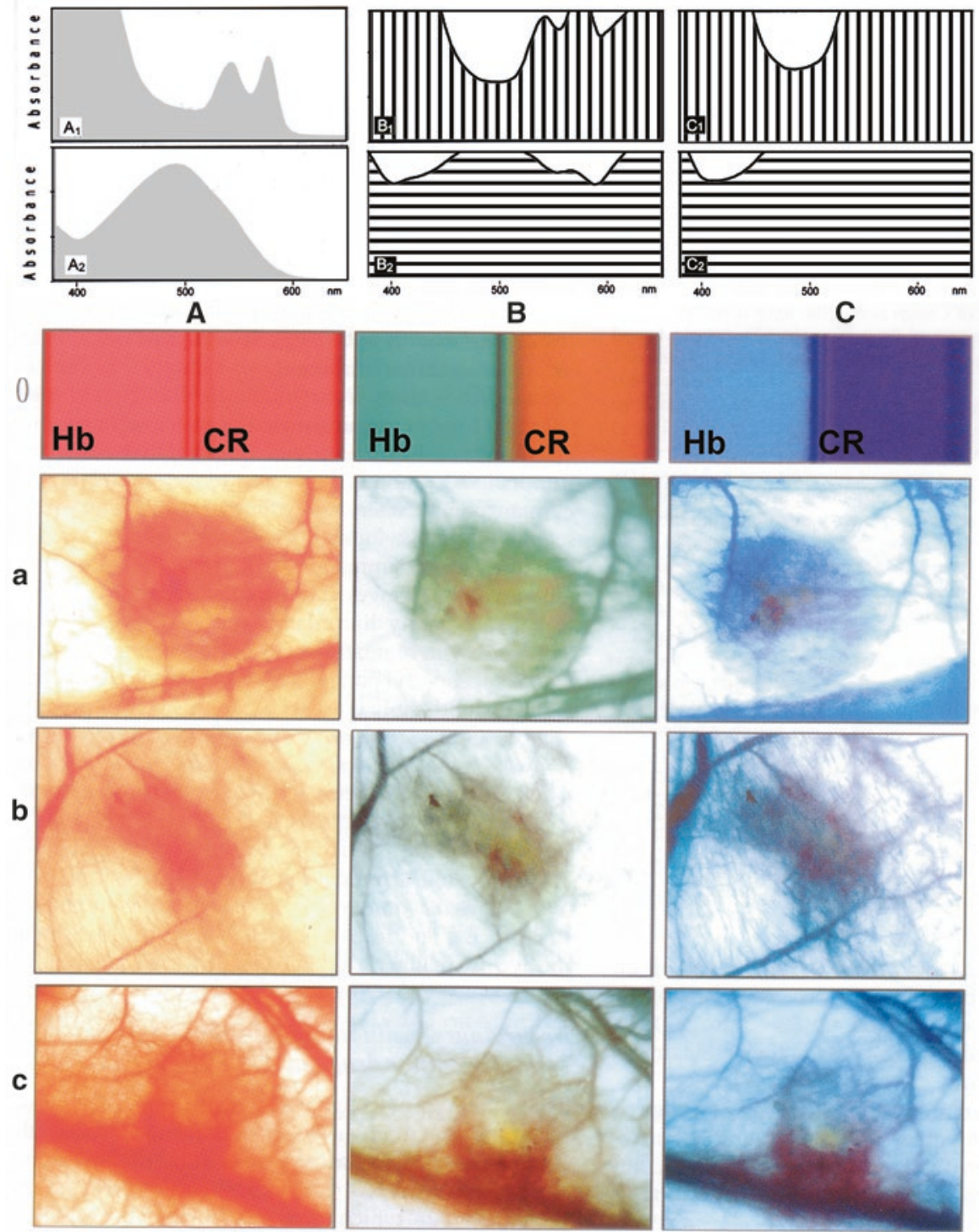

Fig. 2.13 Migration of CR to areas of immune complexation (Arthus reaction induced in rabbitearlobe). Spectroscopic filters depicted above each column were used to differentiate CR and hemoglobin. Column $A$ - spectra of hemoglobin and CR, column $B$ and $C$ - spectra with filters used (4-6) [43] a, b, c - three independent experiments. (Reproduced by permission - Folia Histochemica et Cytobiologica) 

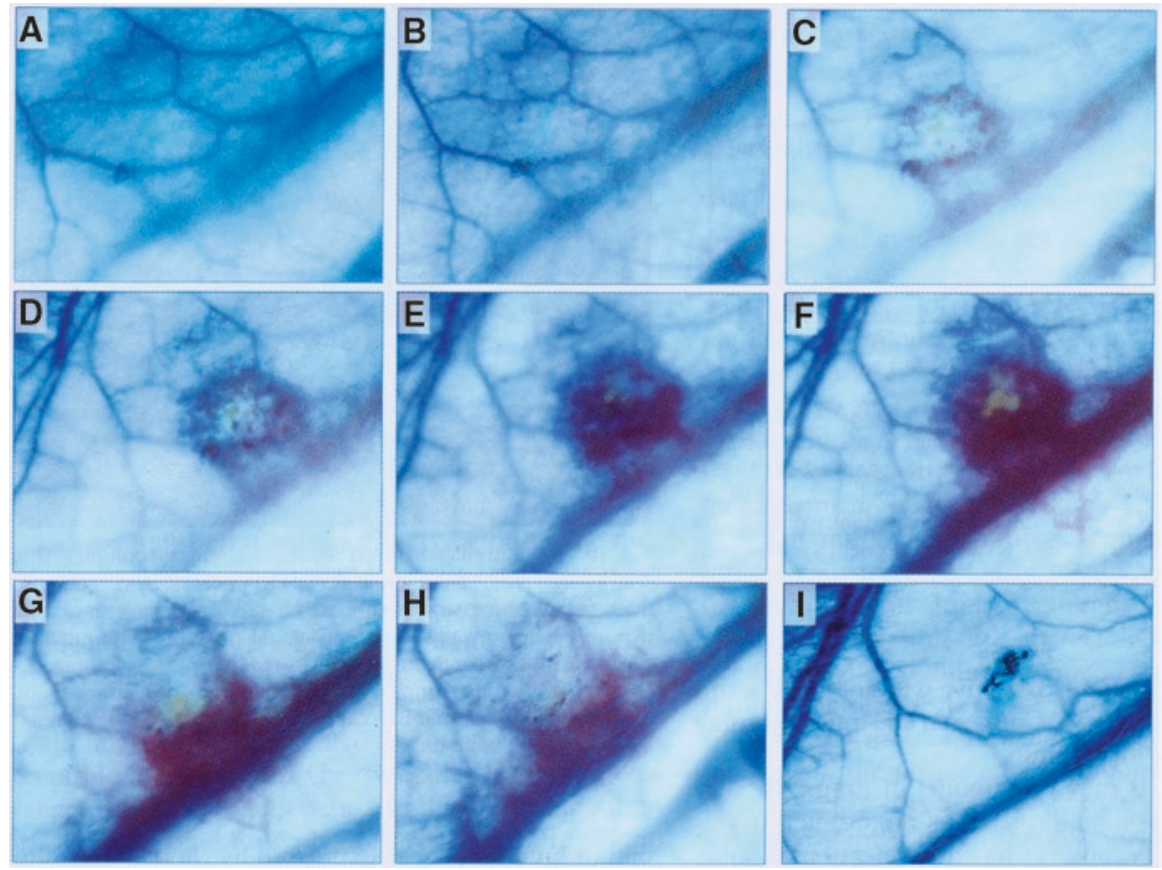

Fig. 2.14 CR accumulation kinetics in the Arthus reaction area: A - 1 h35'; B - 3 h30'; C 4 h35'; D - 5 h $; \mathbf{E}-9$ h30'; F - 26 h30'; G - 52 h35'; H - 72 h20'; I - 14 days (Reproduced by permission - Folia Histochemica et Cytobiologica)

\subsection{Toxicity of CR and Its Applicability for Immunotargeting}

An important practical advantage of CR, as well as of other structurally similar supramolecular systems, is their relatively low toxicity. Supramolecular dye aggregations do not readily penetrate cellular membranes, and are easily excreted, along with the surplus of any intercalated substance (Fig. 2.15). Nevertheless, intestinal excretion of CR has been linked to carcinogenesis - most likely due to bacterial reduction of the dye, producing benzidine (a known carcinogen). This undesirable effect may be mitigated by administering a cellulose-rich diet, since cellulose eagerly binds $\mathrm{CR}$ and protects it from structural changes, including reduction.

In addition to its immune complexation potential, CR is also being studied in the context of amyloidaffinity, although the presented applications of supramolecular 


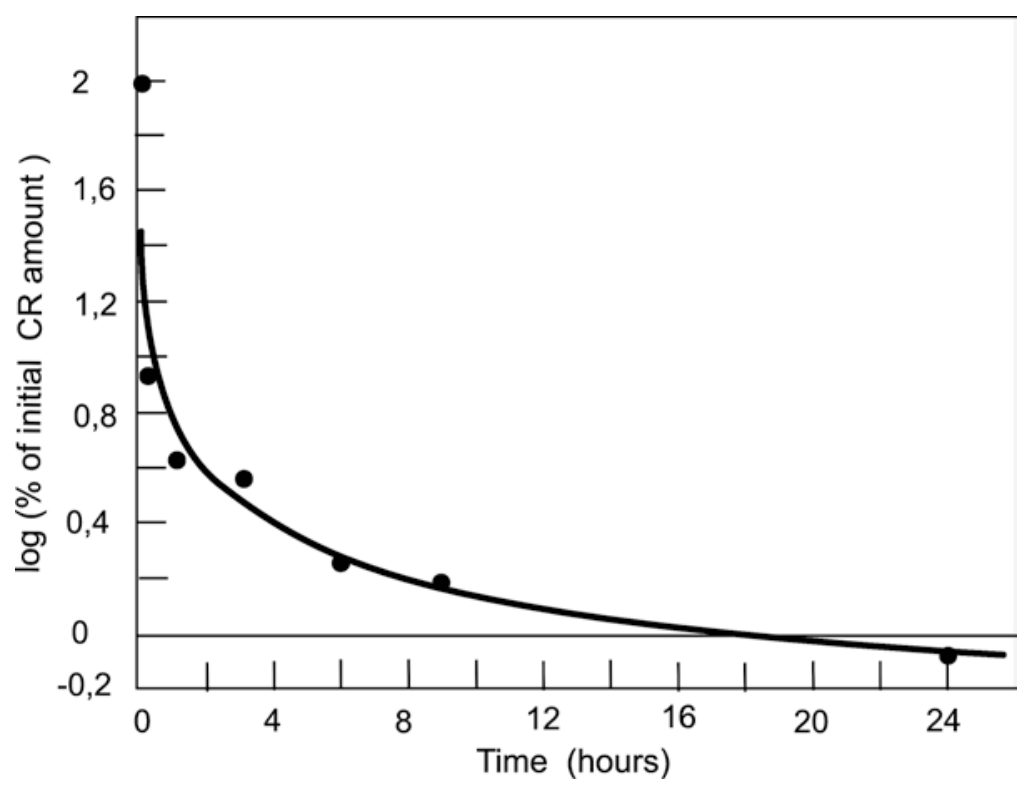

Fig. 2.15 Kinetics of CR clearance from the blood after its intravenous injection ( $2.5 \mathrm{ml}$ of CR $5 \mathrm{mg} / \mathrm{ml}$ ) (Reproduction by permission - Folia Histochemica et Cytobiologica)

ligands are based on laboratory experiments. Practical medical applications would require further, independent research.

Acknowledgements We acknowledge the financial support from the National Science Centre, Poland (grant no. 2016/21/D/NZ1/02763) and from the project Interdisciplinary PhD Studies "Molecular sciences for medicine" (co-financed by the European Social Fund within the Human Capital Operational Programme) and Ministry of Science and Higher Education (grant no. K/ DSC/001370).

\section{References}

1. Edelman GM (1970) The covalent structure of a human gamma G-immunoglobulin. XI Functional implications. Biochemistry 9(16):3197-3205

2. Galvanico NJ, Tomasi TB Jr (1979) Effector sites on antibodies. In: Atassi MZ (ed) Immunochemistry of proteins, vol 3. Plenum Press, New York and London, pp 1-85

3. Brekke OH, Michaelsen TE, Sandlie I (1995) The structural requirements for complement activation by $\mathrm{IgG}$ : does it hinge on the hinge? Immunol Today 16(2):85-90

4. Harris LJ, Larson SB, McPherson A (1999) Comparison of intact antibody structures and the implications for effector function. Adv Immunol 72:191-208 
5. Coloma MJ, Trinh KR, Wims LA, Morrison SL (1997) The hinge as a spacer contributes to covalent assembly and is required for function of IgG. J Immunol 158(2):733-740

6. Cathou RE (1978) Solution conformation an segmental flexibility of immunoglobulins. In: Litman GW, Good RA (eds) Immunoglobulins, vol 5. Plenum Medical Book Company. Comprehensive Immunology, New York/London, pp 37-83

7. Kohoe JM (1978) The structural basis for the biological properties of immunoglobulins. In: Litman GW, Good RA (eds) Immunoglobulins, vol 5. Plenum Medical Book Company, New York/London, pp 173-196

8. Metzger H (1990) General aspects of antibody structure and function. In: Metzger H (ed) Fc receptors and the action of antibodies. American Society Microbiology, Washington, DC, pp 7-11

9. Colman PM, Laver WG, Varghese JN, Baker AT, Tulloch PA, Air GM, Webster RG (1987) Three-dimensional structure of a complex of antibody with influenza virus neuraminidase. Nature 326(6111):358-363

10. Bhat TN, Bentley GA, Fischmann TO, Boulot G, Poljak RJ (1990) Small rearrangements in structures of Fv and Fab fragments of antibody D1.3 on antigen binding. Nature 347(6292):483-485

11. Schulze-Gahmen U, Rini JM, Wilson IA (1993) Detailed analysis of the free and bound conformations of an antibody. X-ray structures of Fab 17/9 and three different Fab-peptide complexes. J Mol Biol 234(4):1098-1118

12. Schneider WP, Wensel TG, Stryer L, Oi VT (1988) Genetically engineered immunoglobulins reveal structural features controlling segmental flexibility. Proc Natl Acad Sci U S A 85(8):2509-2513

13. Sensel MG, Kane LM, Morrison SL (1997) Amino acid differences in the N-terminus of $\mathrm{C}(\mathrm{H}) 2$ influence the relative abilities of $\mathrm{IgG} 2$ and $\mathrm{IgG} 3$ to activate complement. Mol Immunol 34(14):1019-1029

14. Sandlie I, Michaelsen TE (1991) Engineering monoclonal antibodies to determine the structural requirements for complement activation and complement mediated lysis. Mol Immunol 28(12):1361-1368

15. Brekke OH, Michaelsen TE, Sandin R, Sandlie I (1993) Activation of complement by an IgG molecule without a genetic hinge. Nature 363(6430):628-630

16. Rybarska J, Konieczny L, Bobrzecka K, Laidler P (1982) The hemolytic activity of (Fab-Fc) recombinant immunoglobulins with specificity for the sheep red blood cells. Immunol Lett 4(5):279-284

17. Rybarska J, Konieczny L, Roterman I, Piekarska B (1991) The effect of Azo dyes on the formation of immune complexes. Arch Immunol Ther Exp (Warsz) 39:317-327

18. Stopa B, Górny M, Konieczny L, Piekarska B, Rybarska J, Skowronek M et al (1998) Supramolecular ligands: monomer structure and protein ligation capability. Biochimie 80:963-968

19. Kaszuba J, Konieczny L, Piekarska B, Roterman I, Rybarska J (1993) Bis-azo dyes interferencje with effector activation of antibodies. J Physiol Pharmacol 44:233-242

20. Glenner GG, Eanes ED, Page DL (1972) The relation of the properties of Congo red-stained amyloid fibrils to the -conformation. J Histochem Cytochem 20(10):821-826

21. Skowronek M, Stopa B, Konieczny L, Rybarska J, Spólnik P, Zemanek G et al (2003) The structure and protein-binding of amyloid-specific dye reagents. Acta Biochim Pol 50:1213-1227

22. Stopa B, Piekarska B, Konieczny L, Rybarska J, Spólnik P, Zemanek G, Roterman I, Król M (2003) The structure and protein binding of amyloid-specific dye reagents. Acta Biochim Pol 50(4):1213-1227

23. Rybarska J, Piekarska B, Stopa B, Zemanek G, Konieczny L, Nowak M, Król M, Roterman I, Szymczakiewicz-Multanowska A (2001) Evidence that supramolecular Congo red is the 
sole ligation form of this dye for $\mathrm{L}$ chain lambda derived amyloid proteins. Folia Histochem Cytobiol 39(4):307-314

24. Roterman I, No KT, Piekarska B, Kaszuba J, Pawlicki R, Rybarska J, Konieczny L (1993) Bis azo dyes - studies on the mechanism of complex formation with IgG modulated by heating or antigen binding. J Physiol Pharmacol 44(3):213-232

25. Roterman I, Rybarska J, Konieczny L, Skowronek M, Stopa B, Piekarska B et al (1998) Congo red bound to $\alpha-1$ proteinase inhibitor as a model of supramolecular ligand and protein complex. Comput Chem 22:61-70

26. Piekarska B, Konieczny L, Rybarska J, Stopa B, Zemanek G, Szneler E, Król M, Nowak M, Roterman I (2001) Heat-induced formation of a specific binding site for self-assembled Congo red in the $\mathrm{V}$ domain of immunoglobulin L chain lambda. Biopolymers 59(6):446-456

27. Ewert S, Honegger A, Plückthun A (2004) Stability improvement of antibodies for extracellular and intracellular applications: CDR grafting to stable frameworks and structure-based framework engineering. Methods 34(2):184-199

28. Röthlisberger D, Honegger A, Plückthun A (2005) Domain interactions in the Fab fragment: a comparative evaluation of the single-chain $\mathrm{Fv}$ and $\mathrm{Fab}$ format engineered with variable domains of different stability. J Mol Biol 347(4):773-789

29. Smith TJ, Olson NH, Cheng RH, Chase ES, Baker TS (1993) Structure of a human rhinovirusbivalently bound antibody complex: implications for viral neutralization and antibody flexibility. Proc Natl Acad Sci U S A 90(15):7015-7018

30. Thouvenin E, Laurent S, Madelaine MF, Rasschaert D, Vautherot JF, Hewat EA (1997) Bivalent binding of a neutralising antibody to a calicivirus involves the torsional flexibility of the antibody hinge. J Mol Biol 270(2):238-246

31. Pilz I, Kratky O, Licht A, Sela M (1975) Shape and volume of fragments Fab' and (Fab')2 of anti-poly(D-alanyl) antibodies in the presence and absence of tetra-D-alanine as determined by small-angle x-ray scattering. Biochemistry 14(6):1326-1333

32. Piekarska B, Konieczny L, Rybarska J, Stopa B, Spólnik P, Roterman I, Król M (2004) Intramolecular signaling In immunoglobulins - new evidence emerging from the use of supramoelcular proteins ligands. J Physiol Pharmacol 55:487-501

33. Krol M, Roterman I, Drozd A, Konieczny L, Piekarska B, Rybarska J, Spolnik P, Stopa B (2006) The increased flexibility of CDR loops generated in antibodies by Congo red complexation favors antigen binding. J Biomol Struct Dyn 23(4):407-416

34. Jagusiak A, Konieczny L, Król M, Marszałek P, Piekarska B, Piwowar P, Roterman I, Rybarska J, Stopa B, Zemanek G (2015) Intramolecular immunological signal - hypothesis revieved structural background of signalling revealed by Rusing Congo red as a specific tool. Mini Rev Med Chem 14(13):1104-1113

35. Hamada A, Watanabe N, Azuma T, Kobayashi A (1990) Enhancing effect of C1q on IgG monoclonal antibody binding to hapten. Int Arch Allergy Appl Immunol 91(1):103-107

36. Tan LK, Shopes RJ, Oi VT, Morrison SL (1990) Influence of the hinge region on complement activation, C1q binding, and segmental flexibility in chimeric human immunoglobulins. Proc Natl Acad Sci U S A 87(1):162-166. Erratum Proc Natl Acad Sci USA 1991 88, 5066

37. Gaboriaud C, Juanhuix J, Gruez A, Lacroix M, Darnault C, Pignol D, Verger D, FontecillaCamps JC, Arlaud GJ (2003) The crystal structure of the globular head of complement protein C1q provides a basis for its versatile recognition properties. J Biol Chem 278(47):46974-46982

38. Vargas-Madrazo E, Paz-Garcia E (2003) An improved model association for VH-VL immunoglobulin domains: asymmetries between VH and VL in the packing of some interface residues. J Mol Recognit 16:113-120 
39. Skowronek M, Stopa B, Konieczny L, Rybarska J, Piekarska B, Szneler E, Bakalarski G, Roterman I (1998) Self-assembly of Congo red - a theoretical approach to identify its supramolecular organization In water and salt solutions. Biopolymers 46:267-281

40. Król M, Roterman I, Piekarska B, Konieczny L, Rybarska J, Stopa B, Spólnik P, Szneler E (2005) An approach to understand the complexation of supramolecular dye Congo red with immunoglobulin L chain lambda. Biopolymers 77(3):155-162

41. Konieczny L, Piekarska B, Rybarska J, Stopa B, Krzykwa B, Noworolski J, Pawlicki R, Roterman I (1994) Bis azo dye liquid crystalline micelles as possible drug carriers in immunotargeting technique. J Physiol Pharmacol 45(3):441-454

42. Konieczny L, Piekarska B, Rybarska J, Skowronek M, Stopa B, Tabor B, Dabroś W, Pawlicki R, Roterman I (1997) The use of congo red as a lyotropic liquid crystal to carry stains in a model immunotargeting system-microscopic studies. Folia Histochem Cytobiol 35(4):203-210

43. Rybarska J, Piekarska B, Stopa B, Spólnik P, Zemanek G, Konieczny L, Roterman I (2004) In vivo accumulation of self-assembling dye Congo red in an area marked by specific immune complexes: possible relevance to chemotherapy. Folia Histochem Cytobiol 42(2):101-110

Open Access This chapter is distributed under the terms of the Creative Commons Attribution 4.0 International License (http://creativecommons.org/licenses/by/4.0/), which permits use, duplication, adaptation, distribution and reproduction in any medium or format, as long as you give appropriate credit to the original author(s) and the source, provide a link to the Creative Commons license and indicate if changes were made.

The images or other third party material in this chapter are included in the work's Creative Commons license, unless indicated otherwise in the credit line; if such material is not included in the work's Creative Commons license and the respective action is not permitted by statutory regulation, users will need to obtain permission from the license holder to duplicate, adapt or reproduce the material. 